\title{
Clinical utility of a molecular signature in inflammatory demyelinating disease
}

Andrew R. Pachner, MD, Krista DiSano, MS, PhD, Darlene B. Royce, BS, RLATg, and Francesca Gilli, MS, PhD

Neurol Neuroimmunol Neuroinflamm 2019;6:e520. doi:10.1212/NXI.0000000000000520

\section{Abstract}

\section{Objective}

We sought to develop molecular biomarkers of intrathecal inflammation to assist neurologists in identifying patients most likely to benefit from a range of immune therapies.

\section{Methods}

We used Luminex technology and index determination to search for an inflammatory activity molecular signature (IAMS) in patients with inflammatory demyelinating disease (IDD), other neuroinflammatory diagnoses, and noninflammatory controls. We then followed the clinical characteristics of these patients to find how the presence of the signature might assist in diagnosis and prognosis.

\section{Results}

A CSF molecular signature consisting of elevated CXCL13, elevated immunoglobulins, normal albumin CSF/serum ratio $\left(\mathrm{Q}_{\text {albumin }}\right)$, and minimal elevation of cytokines other than CXCL13 provided diagnostic and prognostic value; absence of the signature in IDD predicted lack of subsequent inflammatory events. The signature outperformed oligoclonal bands, which were frequently false positive for active neuroinflammation.

\section{Conclusions}

A CSF IAMS may prove useful in the diagnosis and management of patients with IDD and other neuroinflammatory syndromes.

\section{Classification of evidence}

This study provides Class IV evidence that a CSF IAMS identifies patients with IDD.

\author{
Correspondence \\ Dr. Gilli \\ Francesca.Gilli@dartmouth.edu
}

\section{MORE ONLINE}

$\rightarrow$ Class of Evidence

Criteria for rating

therapeutic and diagnostic

studies

NPub.org/coe 


\section{Glossary}

ADEM = acute disseminated encephalomyelitis; CIS = clinically isolated syndrome; DHMC $=$ Dartmouth-Hitchcock Medical Center; IAMS = inflammatory activity molecular signature; IDD = inflammatory demyelinating diseases; IgG = immunoglobulin G; LP = lumbar puncture; NIND = noninflammatory neurologic diseases; $\mathrm{OCB}=$ oligoclonal bands; OIND = other inflammatory neurologic diseases; OIND-CNS = OIND of the central nervous system; OIND-PNS = OIND of the peripheral nervous system; PPMS = primary progressive MS; RIS = radiologically isolated syndrome; RRMS = relapsing-remitting MS.

The diagnosis and treatment of inflammatory demyelinating diseases (IDD) has become more complex, increasing interest in identifying biomarkers that can assist neurologists. IDD pathology is generally limited to the CNS behind the bloodCSF barrier, and thus, intense effort has been devoted to investigating CSF biomarkers in MS. ${ }^{1-6}$ However, CSF immunoglobulin $\mathrm{G}(\mathrm{IgG})$ abnormalities in MS, first described in $1950{ }^{7}$ remain the only molecular biomarker routinely ordered by neurologists in the clinic, and therefore, a large gap exists in our diagnostic armamentarium. Aiding in the search for IDD biomarkers has been the increased availability of proteomic tools, such as Luminex, a microsphere-based multianalyte processing system allowing simultaneous measurement of dozens of analytes using small input volumes.

Given the availability of a large CSF biobank from patients with neurologic diseases at our institution and an active laboratory using Luminex technology, ${ }^{8,9}$ we pursued the hypothesis that inflammation could be characterized by measuring cytokines and immunoglobulins (Igs) and that this characterization would have clinical utility. In the present study, we investigated 94 patients utilizing Luminex panels for cytokines and Ig subclasses to determine whether these measures could serve as biomarkers for inflammatory activity in IDD. We utilized indexing to CSF and serum albumin concentrations to control for blood-CSF penetration, searching for biomarkers locally produced in the CNS and a molecular signature that would identify distinctive patterns of CNS inflammation in IDD not present in other neuroinflammatory diseases.

We found that patients with active IDD had a pattern on Luminex testing of CSF and serum we labeled the inflammatory activity molecular signature (IAMS), which was helpful prognostically. Patients with inactive forms of IDD, such as many patients with clinically isolated syndrome (CIS), radiologically isolated syndrome (RIS), and primary progressive MS (PPMS), and patients with other neurologic diseases did not have this signature.

\section{Methods}

We sought to develop molecular biomarkers of intrathecal inflammation to assist neurologists in identifying patients most likely to benefit from immunotherapies. This study ultimately provides Class IV evidence that a specific CSF IAMS identifies patients with IDD.

\section{Patients}

During a 24-month period, from September 2015 to September 2017, all patients carrying the diagnosis of IDD who underwent diagnostic lumbar puncture (LP) and gave informed consent for inclusion of their CSF and serum in the Dartmouth-Hitchcock Medical Center's (DHMC) CSF Biobank were included in this study. Patients with other inflammatory neurologic diseases (OIND) and noninflammatory neurologic diseases (NIND) controls were included based on the clarity of their diagnoses, i.e., patients in whom the diagnosis was not clear were excluded. All patients underwent a full diagnostic work-up, including history, examination, routine $\mathrm{CSF} /$ serum analyses, and MRI of the CNS. CSF and serum were banked at $-80^{\circ} \mathrm{C}^{10}$ In 69 patients, CSF and serum were available, and Ig and albumin concentrations were tested in both fluids; in 25 patients, only CSF was available or albumin concentrations were unavailable.

We utilized the revised $2017 \mathrm{McD}$ onald criteria. ${ }^{11}$ Patients were divided into 3 groups (IDD, OIND, and NIND) and 10 subgroups based on their clinical presentation at the time of LP (figure 1): relapsing-remitting MS (RRMS); CIS in which LP was performed within 3 months of the clinical event (CIS-near); CIS in which LP was performed long after the clinical event (CIS-distant); RIS; PPMS; acute disseminated encephalomyelitis (ADEM); OIND of the CNS with high levels of inflammation determined by a total nucleated cell count $>15$ (OIND-CNS-hi-infl); OIND of the CNS with low levels of inflammation determined by a total nucleated cell count $<15$ (OIND-CNS-lo-infl); OIND of the peripheral nervous system (OIND-PNS); and NIND controls. The diagnoses of the CIS group were subsequently adjusted based on the LP results and clinical activity during follow-up. We defined "active" forms of MS similarly to the definition utilized in the McDonald criteria ${ }^{11,12}$; i.e., presence of recent clinical relapses and/or MRI activity (contrast-enhancing lesions, or new or unequivocally enlarging T2 lesions). The conversion status to RRMS based on LP results or subsequent clinical activity is shown in table 1.

The number of patients receiving corticosteroids for their neuroinflammatory event within 3 months of LP were as follows: RRMS (0/4), CIS (25/28), RIS (0/5), PPMS (0/4), and ADEM (2/2). The number of patients on immunomodulatory therapies at the time of their LP were as follows: 


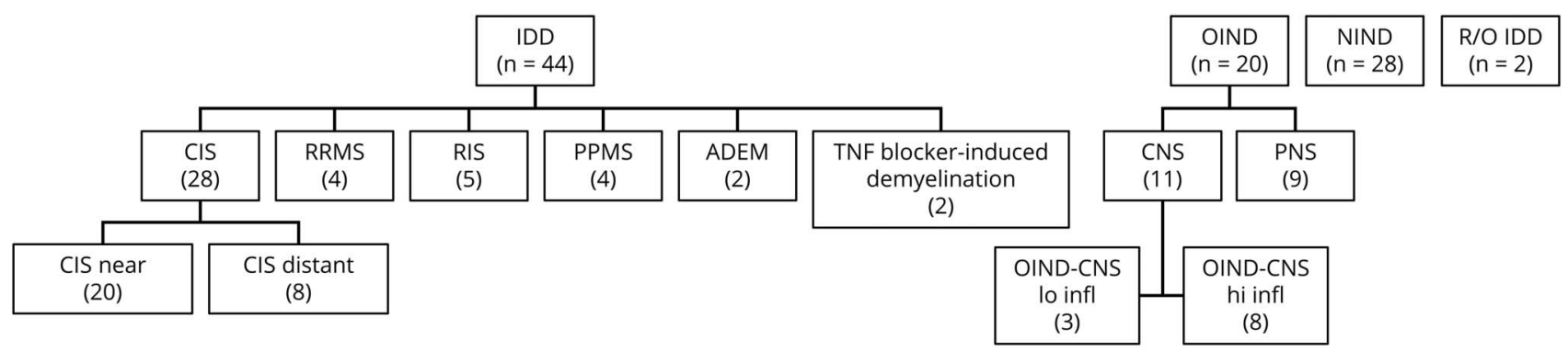

Also included in the study were patients with other neurologic diseases (OIND) and patients with noninflammatory neurologic diseases (NIND) considered controls. Within the IDD group were patients with clinically isolated syndrome (CIS), relapsing remitting MS (RRMS), primary progressive MS (PPMS), acute disseminated encephalomyelitis (ADEM), and demyelination thought to be related to treatment with TNF blockade. The CIS patients were classified as CIS based on information available at the time of the lumbar puncture (LP), and further divided into patients in whom the LP was performed soon after the event (CIS-near) or long after the event (CIS-distant). Within the OIND were patients with OIND of the peripheral nervous system (OIND-PNS) and of the CNS (OINDCNS). Within the latter group were patients with low inflammation (OIND-CNS lo-infl) and high inflammation (OIND-CNS hi-infl). Two patients underwent CSF analysis to rule out IDD, and their diagnosis remains unclear (R/O IDD). All patients underwent LP with CSFs analyzed for routine studies as well as quantitation of cytokines and immunoglobulins by Luminex. Most patients also had similar analysis of serum with determination of indices.

RRMS (3/4), CIS (16/28), RIS (1/5), PPMS (0/4), and $\operatorname{ADEM}(0 / 2)$. The IAMS status of the patients was not significantly affected by these treatments, but numbers in the groups were too small to confidently rule out a treatment effect.

\section{Qalbumin and oligoclonal bands}

Blood-CSF barrier integrity was assessed by measuring albu$\min$ in both fluids. $Q_{\text {albumin, }}[\mathrm{CSF}$ albumin $] /[$ serum albu$\mathrm{min}]$, was calculated based on CSF and serum concentrations determined by Mayo Clinic when an IgG index was ordered on CSF- and serum-matched samples. ${ }^{13,14}$ In our control group, $Q_{\text {albumin }}$ averaged 0.0034 .

Oligoclonal band (OCB) analysis was performed by Mayo Clinic using isoelectric-focusing and immunofixation.

\section{Luminex for immunoglobulins and cytokines}

Cytokine and Ig levels in CSF and serum were measured using the BioplexPro Human Immunoglobulin Isotyping panel and the BioPlexPro Human Chemokine panel (BioRad, Cambridge, MA). These Luminex panels were chosen because the human chemokine/cytokine panel contains 40 molecules commonly altered in inflammatory processes, and the immunoglobulin panel measures separately $4 \mathrm{IgG}$ subclasses as well as $\operatorname{IgM}$ and $\operatorname{IgA}$. Values were expressed in 3 ways:

1. $\mathrm{I}_{\text {analyte }}$ : Where CSF, serum, and $\mathrm{Q}_{\text {albumin }}$ were available, an index was calculated as $\mathrm{I}_{\text {analyte }}=\mathrm{Q}_{\text {analyte }} / \mathrm{Q}_{\text {albumin }}$ with $\mathrm{Q}_{\text {analyte }}=\left[\mathrm{C}_{\text {analyte }}\right.$ in $\left.\mathrm{CSF}\right] /\left[\mathrm{C}_{\text {analyte }}\right.$ in serum $]$; $\mathrm{C}_{\text {analyte }}=$ concentration of analyte.

2. $C_{\text {analyte }}$ If serum or $Q_{\text {albumin }}$ was unavailable, analyte concentration was expressed as $\mathrm{C}_{\text {analyte }}$.

Table 1 Diagnoses of CIS patients at times after the CIS event and subsequent activity in IAMS-negative and IAMS-positive patients

\begin{tabular}{|c|c|c|c|c|c|c|c|}
\hline $\begin{array}{l}\text { Diagnosis prior to } \\
\text { LP }\end{array}$ & $n$ & $\begin{array}{l}\text { LP } \\
\text { timing }\end{array}$ & $\begin{array}{l}\text { Diagnosis after } \\
\text { LP }\end{array}$ & $\begin{array}{l}\text { Activity after } \\
\text { LP }\end{array}$ & $\begin{array}{l}\text { Activity after LP in } \\
\text { IAMS-positive } \\
\text { patients }\end{array}$ & $\begin{array}{l}\text { Activity after LP in } \\
\text { IAMS-negative } \\
\text { patients }\end{array}$ & $\begin{array}{l}\text { Final } \\
\text { diagnosis }\end{array}$ \\
\hline RRMS & 4 & & RRMS & $4 / 4$ & $4 / 4$ & $0 / 0$ & RRMS \\
\hline CIS & 11 & Near & RRMS & $5 / 11$ & $5 / 8$ & $0 / 3$ & RRMS \\
\hline CIS & 5 & Near & $\mathrm{CIS}$ & $0 / 5$ & $0 / 2$ & $0 / 3$ & $\mathrm{CIS}$ \\
\hline CIS & 4 & Near & $\mathrm{CIS}$ & $4 / 4$ & $4 / 4$ & $0 / 0$ & RRMS \\
\hline CIS & 4 & Distant & RRMS & $0 / 4$ & $0 / 0$ & $0 / 4$ & RRMS \\
\hline CIS & 4 & Distant & $\mathrm{CIS}$ & $0 / 4$ & $0 / 1$ & $0 / 3$ & CIS \\
\hline TOT & 32 & & & $13 / 32$ & 13/19 & $0 / 13$ & \\
\hline
\end{tabular}

Abbreviations: CIS = clinically isolated syndrome; IAMS = inflammatory activity molecular signature; LP = lumbar puncture; RRMS = relapsing remitting MS The diagnoses of CIS patients changed during the course of their follow-up after the initial event, according to the 2017 McDonald criteria. ${ }^{11}$ Here, patients are classified according to their diagnosis at the time of the event, i.e., prior to the LP, the diagnosis after results of the CSF analysis, and the final diagnosis after follow-up. Also shown are the IAMS results in each group. The column labeled "activity after LP" represents a summation of the 2 columns "activity after LP in IAMS-positive patients" and "activity after LP in IAMS-negative patients." 


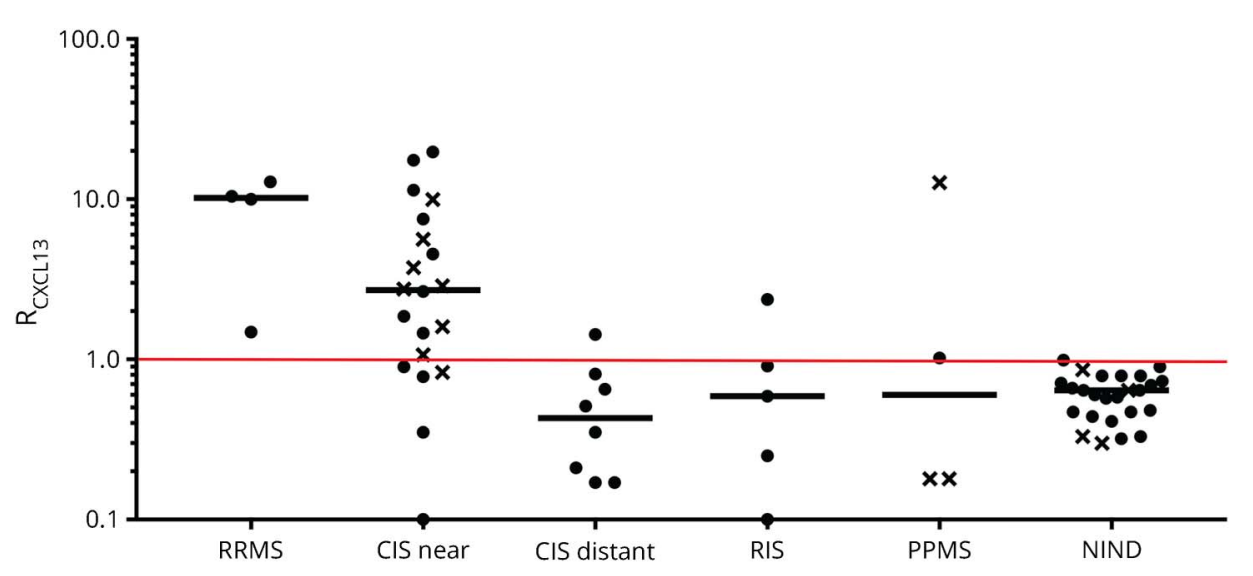

CXCL13 values are expressed as $\mathrm{R}_{\mathrm{CXCL} 13}$ defined by the ratio of $\mathrm{I}_{\mathrm{CXCL13}}$ (circle) or $\mathrm{C}_{\mathrm{CXC13}}(\mathrm{x})$ of the patient to the mean +2 SD of I IXCL13 or $\mathrm{C}_{\mathrm{CXCL} 13}$ of NIND controls. Since the distribution of values for $\mathrm{I}_{\mathrm{CXCL13}}$ and $\mathrm{C}_{\mathrm{CXCL} 13}$ in controls was normal values above 1 were considered significantly elevated above NIND controls. Median $R_{C X C L 13}$ values are shown as a bar. The $\mathrm{R}_{\mathrm{CXC113}}$ calculation was utilized so that the data from complete CSF/serum analyses generating $\mathrm{I}_{\mathrm{CXCL} 13}$ values could be shown using the same $y$-axis as CSF analyses generating $\mathrm{C}_{\mathrm{CXCL13}}$ values. $\mathrm{Pa}$ tient groups were abbreviated as in the legend to figure 1. CIS = clinically isolated syndrome; NIND = noninflammatory neurologic diseases; PPMS = primary progressive MS; RIS = radiologically isolated syndrome; RRMS = relapsing-remitting MS.

3. $\mathrm{R}_{\text {analyte }}$ In figure 2 , values were collated over the multiple runs in which control values were different and values were expressed as $R_{\text {analyte, }}$ defined by the ratio of $\mathrm{I}_{\text {analyte }}$ or $\mathrm{C}_{\text {analyte }}$ of the patient to the mean $+2 \mathrm{SD}$ of $\mathrm{I}_{\text {analyte }}$ or $\mathrm{C}_{\text {analyte }}$ of controls. Values $>1$ are considered significantly elevated above controls.

\section{CSF cell count and protein}

These measurements were performed by the DHMC central laboratory. All the CSFs were atraumatic with RBC counts $<6$ cells.

\section{MRIS}

MRIs of the neuraxis were obtained according to standard practice and were included if performed within 3 months of the LP. The number of gadolinium (Gd)-enhancing lesions was determined by the neuroradiologist and confirmed by a neurologist.

\section{Statistical analysis}

Data from controls were tested for significant deviations from normal distribution $(p<0.05$; D'Agostino and Pearson normality test). Since most cytokines elevated in the CSF of IDD patients, including CXCL13, were normally distributed, values were considered elevated if they were higher than the mean +2 SD of controls; i.e., an $\mathrm{R}$ value $>1$. Correlations were analyzed using Pearson's correlation. Relative risk evaluation was used for testing relationships on categorical variables, e.g., negative and positive IAMS. All statistical analyses were performed by using GraphPad Prism version 7.00 (GraphPad, San Diego, CA).

This study was designed as a nontargeted discovery approach, and, as such, sample sizes for group comparisons were not adequate to achieve statistical power. ${ }^{15}$

\section{Clinical data}

Clinical data were obtained by neurologist review (A.R.P.) of electronic medical records at DHMC.

\section{Data availability}

Anonymized data will be shared upon request by qualified investigators.

\section{Standard protocol approvals, registrations, and patient consents}

Informed consent was obtained from all study participants. The study adhered to the Declaration of Helsinki and was approved by the local ethics committee of DHMC (CPHS\#: STUDY00029241).

\section{Results}

\section{Cytokine and immunoglobulin levels in inflammatory demyelinating disease patients and controls}

Due to the substantial clinical heterogeneity among patients with various types of IDD, we categorized IDD and control patients into groups as shown in figure 1. Sex, age, and CSF characteristics of the patients in these categories are shown in table 2 .

A CSF molecular signature was a consistent feature of IDD patients with inflammatory activity. This signature consisted of:

1. Elevated CSF CXCL13 levels (figure 2)

2. Restricted elevations of other cytokines in the CSF; i.e., levels of $\leq 5$ cytokines elevated other than CXCL13

3. Elevated Ig levels assessed by the Luminex Immuniglobulin panel

4. Normal $Q_{\text {albumin. }}$

\section{Inflammatory demyelinating disease}

Forty-four patients with various forms of idiopathic $\mathrm{IDD}^{16}$ underwent CSF Luminex analysis.

Confirmation of the clinical diagnosis of relapsing-remitting MS In 4 patients with clinical presentations likely to be active RRMS based on history, examination, and MRI findings, an 
Table 2 Patient demographics and CSF profiles

\begin{tabular}{|c|c|c|c|c|c|c|}
\hline Classification (\#n) & $\operatorname{Sex}(F / M)$ & Mean age & Mean TNC & Mean protein & Qalbumin & OCB (+I-) \\
\hline RRMS (n = 4) & $4 / 0$ & $45(30-61)$ & $3(2-4)$ & $39(29-56)$ & $0.0049(0.0027-0.00780$ & $4 / 0$ \\
\hline $\operatorname{CIS}(n=28)$ & $22 / 6$ & $44(18-74)$ & $4(0-11)$ & $33(18-69)$ & $0.0036(0.0017-0.0075)$ & $17 / 6$ \\
\hline RIS (n = 5) & $5 / 0$ & $37(23-49)$ & $2(0-6)$ & $31(24-38)$ & $0.0040(0.0025-0.0060)$ & $3 / 2$ \\
\hline PPMS $(n=4)$ & $2 / 2$ & $48(40-61)$ & $8(0-31)$ & $34(24-50)$ & $0.0038(0.0022-0.0054)$ & $4 / 0$ \\
\hline ADEM $(n=2)$ & $1 / 1$ & $35(21-56)$ & $59(0-10)$ & $36(25-47)$ & $0.0058(0.0030-0.0086)$ & $1 / 1$ \\
\hline Post-TNF blocker $(n=1)$ & $1 / 0$ & 39 & 0 & 31 & ND & $1 / 0$ \\
\hline OIND-CNS (hi infl) $(n=7)$ & $3 / 4$ & $57(39-66)$ & $69(19-137)$ & $90(48-148)$ & $0.0137(0.0067-0.0187)$ & $4 / 3$ \\
\hline OIND-CNS (lo infl) $(n=4)$ & $2 / 2$ & $34(22-39)$ & $4(0-11)$ & $38(23-77)$ & $0.0034(0.0029-0.0039)$ & $3 / 1$ \\
\hline OIND-PNS (n = 9) & $8 / 1$ & $66(49-81)$ & $1(0-3)$ & $80(42-198)$ & $0.0147(0.0091-0.0392)$ & $1 / 6$ \\
\hline NIND $(n=28)$ & $18 / 10$ & $46(20-64)$ & $1(0-4)$ & $30(17-57)$ & $0.0040(0.0022-0.0055)$ & $0 / 25$ \\
\hline
\end{tabular}

Abbreviations: $A D E M=$ acute disseminated encephalomyelitis; $C I S=$ clinically isolated syndrome; ND = not done; NIND = noninflammatory neurologic diseases; OCB = oligoclonal bands; OIND-PNS = OIND of the peripheral nervous system; PPMS = primary progressive MS; RIS = radiologically isolated syndrome; RRMS = relapsing-remitting MS; TNC = total nucleated cells.

Patient groups were abbreviated as in legend to figure 1. In the OIND-CNS, the "hi infl" had >15 TNCs per $\mu$ L in their CSF, while "lo infl" had less than 15 TNCs per $\mu \mathrm{L}$. Numbers in parentheses represent the range of the values.

LP was performed to confirm the diagnosis. None of these patients had a relapse or Gd-enhancing lesion within 3 months before or after the LP. IAMS was found to be positive in all 4 patients, with other cytokine indices elevated in this group, including CXCL10 (2/4), CCL3, CXCL8, IL10, IL16, and CXCL11 (1/4), although none of the RRMS patients had $>3$ elevated cytokines. All 4 patients had activity within mean of 2.1 years of follow-up after their LP.

\section{Clinically isolated syndrome}

Twenty-eight patients underwent an LP to aid in the evaluation of a first clinical episode meeting the $2017 \mathrm{McD}$ onald criteria for CIS. ${ }^{11}$ Both CSF and serum were available for 16 patients; for the other 12 patients, only CSF was tested.

In 20 of these patients, the time from onset of the symptoms of CIS to the LP was relatively short, 2.6 months (range: $0.3-5.8$ months). These spinal taps were performed to better define the acute event, and the range of times was usually due to variability of patient acceptance of the procedure. Although the inflammatory milieu could have quieted within that time frame, there was no correlation, positive or negative, between presence of IAMS and time after LP. In a mean period of 2.2 years after the CIS event, 9 of 20 patients developed new activity. All of these patients had a positive IAMS. In contrast, all 6 of the CIS-near patients without IAMS did not develop new activity. A positive IAMS did not definitively predict future activity, since 5 of the CIS-near patients had a positive IAMS without subsequent activity. Since the follow-up period was relatively short, IAMS positivity in these patients may indicate that during a longer follow-up period they would have eventually developed new activity.
Eight of the 28 patients diagnosed as having experienced CIS underwent an LP more than 4 years (mean: 8.9 years and range: 4.5-11.7 years) after an acute, demyelinating neurologic event and were grouped as "CIS-distant". LP was performed to evaluate whether nonspecific symptoms such as headache, seizures, or severe fatigue represented new events consistent with MS or symptoms unrelated to the CIS event. Four of 8 of these patients were OCB positive, all of whom had elevated $\mathrm{I}_{\mathrm{Ig}}$ or $\mathrm{C}_{\mathrm{Ig}}$. However, only 1 of 8 patients had a positive IAMS, and this patient was diagnosed by brain biopsy as having a grade 3 oligodendroglioma. The ultimate diagnoses, OCB positivity and IAMS status of the patients with CIS are shown in tables 1 and 3.

\section{Primary progressive MS}

In 4 patients with relatively little disability, diagnosis of PPMS was felt to be likely, but progression was slow, and LP was performed to confirm the diagnosis. All 4 were OCB positive. Three of the 4 patients (PPMS-lo infl), with an average age of 54 years, had no enhancing lesions on MRI of the brain or spinal cord and were negative for IAMS because of normal $\mathrm{I}_{\mathrm{CXCL13}}$ values. In contrast, a 41-year-old patient with enhancing lesions in the spinal cord at the time of the LP (PPMS-hi infl) had a positive IAMS with elevated $\mathrm{I}_{\mathrm{CXCL13}}$ and additionally, elevated CXCL9, CXCL11, and CCL22.

\section{Radiologically isolated syndrome}

Five patients fulfilled criteria for RIS. ${ }^{17}$ In a follow-up averaging 1.6 years (range: $0.5-2.8$ years) after the abnormal MRI, none of these patients developed an attack or new MRI lesions. Three of the 5 patients had positive OCBs. One patient had a positive IAMS, a 37-year-old woman with nonspecific symptoms of headache and fatigue. A history of severe 
Table 3 OCB positivity vs IAMS positivity

\begin{tabular}{|c|c|c|c|c|c|}
\hline Diagnosis prior to LP & $\mathbf{N}$ & Diagnosis after LP & Final diagnosis after follow-up & OCB positivity & IAMS positivity \\
\hline RRMS & 4 & RRMS & RRMS & $100 \%$ & $100 \%$ \\
\hline \multirow[t]{3}{*}{ CIS (near) } & 11 & RRMS & RRMS & $100 \%$ & $73 \%$ \\
\hline & 4 & CIS & RRMS & 0 & $100 \%$ \\
\hline & 5 & CIS & CIS & 0 & $60 \%$ \\
\hline \multirow[t]{2}{*}{ CIS (distant) } & 4 & RRMS & RRMS & $100 \%$ & 0 \\
\hline & 4 & CIS & CIS & 0 & $25 \%^{\mathrm{a}}$ \\
\hline RIS & 5 & RIS & RIS & $60 \%$ & 0 \\
\hline PPMS (lo-infl) & 3 & PPMS & PPMS & $100 \%$ & 0 \\
\hline PPMS (hi-infl) & 1 & PPMS & PPMS & $100 \%$ & $100 \%$ \\
\hline ADEM & 2 & ADEM & ADEM & $50 \%$ & 0 \\
\hline OIND-CNS (hi-infl) & 7 & OIND-CNS & OIND-CNS & $57 \%$ & 0 \\
\hline OIND-CNS (lo-infl) & 4 & OIND-CNS & OIND-CNS & $75 \%$ & $100 \%$ \\
\hline OIND-PNS & 9 & OIND-PNS & OIND-PNS & $22 \%$ & 0 \\
\hline NIND & 28 & NIND & NIND & 0 & 0 \\
\hline
\end{tabular}

Abbreviations: $\mathrm{ADEM}$ = acute disseminated encephalomyelitis; $\mathrm{CIS}=$ clinically isolated syndrome; IAMS = inflammatory activity molecular signature; $\mathrm{ND}$ = not done; NIND = noninflammatory neurologic diseases; OCB = oligoclonal bands; OIND-PNS = OIND of the peripheral nervous system; PPMS = primary progressive MS; RIS = radiologically isolated syndrome; RRMS = relapsing-remitting MS.

Patient groups were abbreviated as in legend to figure 1. Groups are identical to those reported in table 1.

a One patient with a grade 3 oligodendroglioma.

MS in the mother and a sister of the patient led her primary care physician to order an MRI of the brain, which revealed multifocal white matter disease consistent with demyelinating disease, but without any enhancing lesions. In a follow-up of 1.2 years, there was no clinical or radiologic change.

\section{Acute disseminated encephalomyelitis}

Two patients fulfilled criteria for ADEM, ${ }^{18,19}$ both with a monophasic, highly inflammatory event with $>10$ Gdenhancing lesions in brain and spinal cord and no new clinical or radiologic events after 2.6 and 2.9 years of follow-up. The CSF was obtained during the acute neuroinflammation. One CSF was OCB negative and the other was OCB positive. Neither had positive IAMS; both had elevated $Q_{\text {albumin }}$ and Ig indices but normal indices for CXCL13 and other cytokines.

\section{TNF blocker-induced demyelination}

A 39-year-old woman had IDD thought to be secondary to treatment of autoimmune uveitis with adalimumab and, after 3.5 years of follow-up after the event, had no new demyelinating events off of the TNF blocker. She had negative IAMS, positive OCBs, and elevated $\mathrm{C}_{\mathrm{Ig}}$ at the time of her myelitis, but normal CSF concentrations of all cytokines, including CXCL13.

\section{Confirmation of the absence of MS}

In 2 patients referred to DHMC with the diagnosis of MS, but in whom the diagnosis was felt to be incorrect, CSF was obtained for diagnostic purposes. One of these patients, who was OCB negative, is currently being followed with the clinical impression of nonspecific or nonlocalizing neurologic symptoms with abnormal MRI. ${ }^{20}$ The other patient was OCB positive and turned out to have a glioblastoma. Both patients were IAMS negative.

\section{Correlation between inflammatory activity molecular signature and contrast-enhancing lesions}

Twenty-seven of the 36 CIS, RRMS, and PPMS patients underwent MRI scanning within 3 months of LP. As expected, MRIs within 3 months of LP tended to be performed on more active patients. Thus, 17 of 27 patients had at least 1 enhancing lesion $($ mean $=4)$. In these relatively active patients, 3 of 10 patients without Gd-enhancing lesion had negative IAMS, whereas only 1 of 17 patients with Gd-enhancing lesions had negative IAMS. Using a relative risk analysis, patients without Gd-enhancing lesions were shown to be 5.1 times as likely as patients with Gd-enhancing lesions to have a negative IAMS.

\section{Other nondemyelinating inflammatory neurologic diseases}

Eleven patients in the study were classified as OIND-CNS There were 7 patients with neuroinfections or neurosarcoidosis, i.e., Lyme meningitis $(n=2)$, viral meningitis or meningoencephalitis $(n=3)$, neurocysticercosis $(n=1)$, and neurosarcoidosis $(n=1)$, and there were 4 patients with less 
inflammatory disease, i.e., toxoplasma encephalitis and HIV positivity, neuropsychiatric systemic lupus erythematosus, Sjogren's CNS involvement, and low-grade viral meningitis. Seven HIV-negative patients with CNS infections or neurosarcoidosis had a distinctive CSF pattern with high values for CSF total nucleated cells, elevated $\mathrm{Q}_{\text {albumin, }}$ and elevations in a broad range of cytokines. For these patients, the average number of CSF cytokine levels above the cutoff (mean +2 SD of NIND controls) was 34.2 of the 39 cytokines tested. Four of 7 had positive OCB. The 4 less inflammatory patients had only a few cytokine elevations. Three of these 4 patients had a positive IAMS, but their clinical picture, including lack of demyelination, resulted in no confusion with IDD. All 11 patients in the OIND-CNS group had elevated CXCL13 levels. Two of the highest values for $\mathrm{I}_{\mathrm{CXCL} 13}$ were in Lyme meningitis patients.

Nine patients had inflammatory PNS disease (OIND-PNS), none of whom had positive IAMS. Four had chronic inflammatory demyelinating polyneuropathy, 2 had acute inflammatory demyelinating polyradiculoneuropathy, and 1 each had vasculitic neuropathy, HIV neuropathy, and sarcoid neuropathy. None of these patients had increased total nucleated cells, while all had elevated protein. In all the 5 patients in whom $\mathrm{Q}_{\text {albumin }}$ was tested, this parameter was elevated. In 7 patients in whom OCBs were tested, only 1 was positive.

\section{Noninflammatory neurologic disease controls}

All patients underwent LP as part of a diagnostic evaluation for neurologic symptoms. Thus, none of our controls were truly "normal," but we considered 28 patients with a confirmed diagnosis that was clear and noninflammatory as appropriate controls and labeled the group NIND. Thirteen patients had noninflammatory headache syndromes, including idiopathic intracranial hypertension or migraine, 4 had idiopathic epilepsy, 5 had noninflammatory neuropathies, 4 had brain white matter disease attributable to microvascular disease, and 2 had Alzheimer's disease. CSF from these patients had normal standard analysis, normal $Q_{\text {albumin, were }}$ negative for OCBs or $\mathrm{I}_{\mathrm{Ig}}$, and had CXCL13 concentrations/ indices within the normal range.

\section{Correlation of $\mathbf{R}_{\mathrm{CXCL} 13}$ with CSF measures and imaging}

The number of Gd-enhancing lesions tended to be higher in patients presenting with higher $\mathrm{R}_{\mathrm{CXCL13}}$ values, with the mean $\mathrm{R}_{\mathrm{CXCL13}}$ for 8 patients with $>1$ enhancing lesions being 15 and for 19 patients with 0 or 1 enhancing lesion being 6; however, this was not statistically significant. Similar correlations were seen for CSF TNCs, OCB number, and CSF IgG1 concentration, but none were statistically significant. There was no correlation between $\mathrm{R}_{\mathrm{CXCL13}}$ and OCB positivity.

\section{Qalbumin as a measure of blood-CSF barrier integrity}

As previously noted, ${ }^{13} \mathrm{Q}_{\text {albumin }}$ in IDD patients was not different from controls (0.0039 for RRMS/PPMS/CIS/RIS patients compared to 0.0043 for controls). The range of values was wide for IDD patients (0.0017-0.0085) and approximately the same in controls. Inflammatory diseases other than IDD had high $Q_{\text {albumin }}$ with the OIND-PNS and OIND-CNS-hi-infl average $\mathrm{Q}_{\text {albumin }}$ levels of 0.0147 and 0.0137 .

\section{Evidence of constitutive production of cytokines}

The majority of cytokine indices ranged between 3 and 30, a range that could be predicted from the $8-10 \mathrm{kDa}$ molecular weight of these proteins. ${ }^{5}$ However, some mean cytokine indices in the controls were over 500, such as CXCL10, CCL2, CXCL16, and CXCL12, providing evidence for constitutive intrathecal production.

\section{Discussion}

This article identifies a distinctive CSF signature of active inflammation in IDDs utilizing Luminex technology and validates the signature's clinical utility both longitudinally and cross-sectionally. The combination of (1) elevated CXCL13 index; (2) restricted elevations of other cytokine indices; (3) elevated Ig as manifested by OCBs or Ig indices; and (4) normal $Q_{\text {albumin }}$ values was unique to CIS and active forms of MS and not seen in the other neuroinflammatory diseases tested, i.e., ADEM, inactive PPMS, or non-demyelinating neuroinflammatory diseases. This signature is helpful in identifying activity in IDD but is not a useful diagnostic assay for MS due to false negatives, e.g., 3 of 4 patients with PPMS, and false positives, e.g., other inflammatory neurologic disease with mild CNS inflammation.

The use of indices for most of our analysis was important, correcting for serum values and blood-CSF barrier disruption. When we established our CSF biobank, we collected CSF samples alone, as done in most previous CSF cytokine studies in IDD, ${ }^{1-3,21-23}$ with occasional concurrent serum samples. However, it became clear that measuring CSF alone would not be sufficient since many cytokine serum levels were quite variable and blood-CSF integrity $\left(Q_{\text {albumin }}\right)$ was also variable. This variability resulted in wide swings in CSF analyte concentrations, independent of its intrathecal production. For instance, serum CXCL13 concentrations ranged from 27 to $243 \mathrm{pg} / \mathrm{mL}$, a nine-fold difference. $Q_{\text {albumin }}$ ranged from 0.0017 to 0.0085 , a five-fold difference, even within the relatively narrow range of IDD and controls. Thus, fluctuation in these 2 variables could be as high as 45 -fold but were unrelated to intrathecal production. A few other investigators recently have recognized the importance of correcting for these variables in assessing CSF biomarkers. ${ }^{5,24}$ Thus, the majority of the cytokine data in this study is based on $\mathrm{I}_{\text {analyte, }}$ with a raised index being strong supportive evidence of intrathecal production of the analyte. ${ }^{13}$ Albumin has been the choice of reference molecule for calculation of CSF indices ${ }^{25}$ because it is produced exclusively in the liver and because of its intermediate size of $66 \mathrm{kD}$. 
The potential application for IAMS most likely to be clinically useful is in predicting the likelihood of future activity. In 26 patients, 13 CIS, 4 PPMS, 5 RIS, 2 ADEM, and 2 possible MS, a negative IAMS predicted lack of activity during the mean 2.3 years after LP. There were no patients with negative IAMS who developed activity during this period. The IAMS result may inform treatment decisions as a clinician might consider not initially using disease-modifying therapy on patients with negative IAMS.

As others have noted, ${ }^{26,27}$ elevated CXCL13 levels are not specific for MS. High CXCL13 levels are a consistent feature of Borrelia burgdorferi infection, ${ }^{28}$ and 2 of the highest $\mathrm{I}_{\mathrm{CXCL} 13}$ levels in our study were in patients with Lyme neuroborreliosis. Eight of 11 patients in our OIND-CNS group had elevated CSF CXCL13, but patients had profiles very different than the IDD and control groups, with broad cytokine elevations and very high levels of $Q_{\text {albumin }}$ (average of 0.0123 vs 0.004 in IDD and control groups).

The source of intrathecal CXCL13 production in IDDs has not been definitively determined. Possibilities include macrophage lineage cells ${ }^{29}$ or microglia, ${ }^{30}$ plasmacytoid dendritic cells, ${ }^{31}$ astrocytes, ${ }^{32}$ or follicular dendritic cells. ${ }^{33}$ Some CXCL13 production may occur within specially organized lymphoid tissue called ectopic lymphoid follicles in MS, mostly located in the meninges, ${ }^{34-36}$ but selective intraparenchymal induction of CXCL13 within active MS plaques has also been described. ${ }^{37}$

We did not find positive OCBs to be as helpful as IAMS in predicting future activity. $\mathrm{OCBs}$ or increased $\mathrm{I}_{\mathrm{Ig}}$ in the absence of the remaining IAMS-positive components appeared to indicate that active inflammation was present at some point during the course of illness, likely during the neuroinflammatory episode defining CIS, but not necessarily at the time of LP. In our study, 5 of 8 patients with CIS who had not converted to MS an average of 8.9 years after CIS were OCB positive, indicating a high rate of false positivity for $\mathrm{OCB}$ as a measure of active inflammation, a finding consistent with a recent meta-analysis. ${ }^{38}$ In addition, 4 of 4 patients with PPMS in our study were OCB positive, with only 1 with a positive IAMS showing evidence of active inflammation. It is possible that CNS microenvironments providing local availability of B-cell permissive factors, including CXCL10, BAFF, and APRIL, ${ }^{39,40}$ can establish antibody-secreting cell "niches" within the CNS following transient inflammation without the need for recurrent inflammation. In contrast, persistent CXCL13 production within the CNS may require ongoing inflammation.

Our study suffers from some limitations. First, there were relatively low numbers in each group as 44 IDD patients were divided into 7 clinical categories. Second, for those diagnosed with CIS, there was a limited follow-up after the LP. Third, cutoffs for positivity were based on concurrently analyzing numerous noninflammatory controls; thus, for this test to be clinically helpful, absolute values for cutoffs will need to be determined to minimize the number of negative controls that will be required. Fourth, 25 patients did not have full index determinations because either serum or albumin determinations were unavailable. Finally, because an LP is rarely performed on secondary progressive MS patients in clinical practice, this MS patient subgroup was not included in the study, despite evidence that some of these patients demonstrate active intrathecal inflammation. ${ }^{6}$ Considering these limitations, the clinical value of IAMS in IDD will have to be confirmed in a prospective trial.

Our study identifies CSF molecular signature as a powerful biomarker for the distinctive intrathecal inflammation in IDDs, which may have clinical utility for neurologists faced with difficult management challenges in inflammatory neurologic diseases.

\section{Author contributions}

All authors contributed to study concept and design and to data acquisition and analysis; A.R. Pachner, K. DiSano, and F. Gilli contributed to drafting the manuscript and figures.

\section{Acknowledgment}

The authors thank Emily Clough for her excellent administrative assistance.

\section{Study funding}

This research was funded by research grants from EMDSerono, Bornstein Research Fund, Diamond endowment, Edgerton Fund, and the Hitchcock Foundation.

\section{Disclosure}

A.R. Pachner received research support from EMD-Serono, Genzyme, Roche, Novartis, and Biogen. K. DiSano and D.B. Royce report no disclosures. F. Gilli received research support from Hitchcock Foundation. Full disclosure form information provided by the authors is available with the full text of this article at Neurology.org/NN.

Received May 22, 2018. Accepted in final form October 2, 2018.

\section{References}

1. Alvarez E, Piccio L, Mikesell RJ, et al. CXCL13 is a biomarker of inflammation in multiple sclerosis, neuromyelitis optica, and other neurological conditions. Mult Scler 2013;19:1204-1208.

2. Bielekova B, Komori M, Xu Q, Reich DS, Wu T. Cerebrospinal fluid IL-12p40, CXCL13 and IL-8 as a combinatorial biomarker of active intrathecal inflammation. PloS one 2012; 7:e48370.

3. Novakova L, Axelsson M, Khademi M, et al. Cerebrospinal fluid biomarkers as a measure of disease activity and treatment efficacy in relapsing-remitting multiple sclerosis. J Neurochem 2017;141:296-304.

4. Teunissen CE, Dijkstra C, Polman C. Biological markers in CSF and blood for axonal degeneration in multiple sclerosis. Lancet Neurol 2005;4:32-41.

5. Edwards KR, Goyal J, Plavina T, et al. Feasibility of the use of combinatorial chemokine arrays to study blood and CSF in multiple sclerosis. PLoS One 2013;8:e81007.

6. Komori M, Blake A, Greenwood M, et al. Cerebrospinal fluid markers reveal intrathecal inflammation in progressive multiple sclerosis. Ann Neurol 2015;78:3-20.

7. Kabat EA, Freedman DA, Murray JP, Knaub V. A study of the crystalline albumin, gamma globulin and total protein in the cerebrospinal fluid of 100 cases of multiple sclerosis and in other diseases. Am J Med Sci 1950;219:55-64.

8. Gilli F, Li L, Pachner AR. The immune response in the CNS in Theiler's virus induced demyelinating disease switches from an early adaptive response to a chronic innatelike response. J Neurovirol 2016;22:66-79. 
9. Gilli F, Royce DB, DiSano KD, Pachner A. Treatment of Theiler's virus-induced demyelinating disease with teriflunomide. J Neurovirol 2017;23:825-838.

10. Teunissen CE, Tumani H, Engelborghs S, Mollenhauer B. Biobanking of CSF: international standardization to optimize biomarker development. Clin Biochem 2014; 47:288-292.

11. Thompson AJ, Banwell BL, Barkhof F, et al. Diagnosis of multiple sclerosis: 2017 revisions of the McDonald criteria. Lancet Neurol 2018;17:162-173.

12. Lublin FD, Reingold SC, Cohen JA, et al. Defining the clinical course of multiple sclerosis: the 2013 revisions. Neurology 2014;83:278-286.

13. Reiber H. Cerebrospinal fluid-physiology, analysis and interpretation of protein patterns for diagnosis of neurological diseases. Mult Scler 1998;4:99-107.

14. Reiber H, Peter JB. Cerebrospinal fluid analysis: disease-related data patterns and evaluation programs. J Neurol Sci 2001;184:101-122.

15. Anderson DC, Kodukula K. Biomarkers in pharmacology and drug discovery. Biochem Pharmacol 2014;87:172-188.

16. Miller DH, Weinshenker BG, Filippi M, et al. Differential diagnosis of suspected multiple sclerosis: a consensus approach. Mult Scler 2008;14:1157-1174.

17. Okuda DT. Radiologically isolated syndrome: MR imaging features suggestive of multiple sclerosis prior to first symptom onset. Neuroimaging Clin N Am 2017;27: 267-275.

18. Krupp LB, Tardieu M, Amato MP, et al. International Pediatric Multiple Sclerosis Study Group criteria for pediatric multiple sclerosis and immune-mediated central nervous system demyelinating disorders: revisions to the 2007 definitions. Mult Scler 2013;19:1261-1267.

19. Pohl D, Alper G, Van Haren K, et al. Acute disseminated encephalomyelitis: updates on an inflammatory CNS syndrome. Neurology 2016;87:S38-S45.

20. Solomon AJ, Bourdette DN, Cross AH, et al. The contemporary spectrum of multiple sclerosis misdiagnosis: a multicenter study. Neurology 2016;87:1393-1399.

21. Fitzner B, Hecker M, Zettl UK. Molecular biomarkers in cerebrospinal fluid of multiple sclerosis patients. Autoimmun Rev 2015;14:903-913.

22. Housley WJ, Pitt D, Hafler DA. Biomarkers in multiple sclerosis. Clin Immunol 2015; 161:51-58.

23. Modvig S, Degn M, Horwitz H, et al. Relationship between cerebrospinal fluid biomarkers for inflammation, demyelination and neurodegeneration in acute optic neuritis. PloS one 2013;8:e77163.

24. Alvarez E, Piccio L, Mikesell RJ, et al. Predicting optimal response to B-cell depletion with rituximab in multiple sclerosis using CXCL13 index, magnetic resonance imaging and clinical measures. Mult Scler J Exp Transl Clin 2015;1:2055217315623800.

25. Link H, Tibbling G. Principles of albumin and IgG analyses in neurological disorders. III. Evaluation of IgG synthesis within the central nervous system in multiple sclerosis. Scand J Clin Lab Invest 1977;37:397-401.
26. Khademi M, Kockum I, Andersson ML, et al. Cerebrospinal fluid CXCL13 in multiple sclerosis: a suggestive prognostic marker for the disease course. Mult Scler 2011;17: 335-343.

27. Kowarik MC, Cepok S, Sellner J, et al. CXCL13 is the major determinant for B cell recruitment to the CSF during neuroinflammation. J Neuroinflammation 2012;9:93.

28. Pachner AR, Dail D, Narayan K, Dutta K, Cadavid D. Increased expression of B-lymphocyte chemoattractant, but not pro-inflammatory cytokines, in muscle tissue in rhesus chronic Lyme borreliosis. Cytokine 2002;19:297-307.

29. Carlsen HS, Baekkevold ES, Morton HC, Haraldsen G, Brandtzaeg P. Monocyte-like and mature macrophages produce CXCL13 (B cell-attracting chemokine 1) in inflammatory lesions with lymphoid neogenesis. Blood 2004;104:3021-3027.

30. Rainey-Barger EK, Rumble JM, Lalor SJ, Esen N, Segal BM, Irani DN. The lymphoid chemokine, CXCL13, is dispensable for the initial recruitment of B cells to the acutely inflamed central nervous system. Brain Behav Immun 2011;25:922-931.

31. Narayan K, Dail D, Li L, et al. The nervous system as ectopic germinal center: CXCL13 and IgG in lyme neuroborreliosis. Ann Neurol 2005;57:813-823.

32. Lokensgard JR, Mutnal MB, Prasad S, Sheng W, Hu S. Glial cell activation, recruitment, and survival of B-lineage cells following MCMV brain infection. J Neuroinflammation 2016;13:114.

33. Serafini B, Rosicarelli B, Magliozzi R, Stigliano E, Aloisi F. Detection of ectopic B-cell follicles with germinal centers in the meninges of patients with secondary progressive multiple sclerosis. Brain Pathol 2004;14:164-174.

34. Magliozzi R, Howell O, Vora A, et al. Meningeal B-cell follicles in secondary progressive multiple sclerosis associate with early onset of disease and severe cortical pathology. Brain 2007;130:1089-1104.

35. Aloisi F, Columba-Cabezas S, Franciotta D, et al. Lymphoid chemokines in chronic neuroinflammation. J Neuroimmunol 2008;198:106-112.

36. Lalor SJ, Segal BM. Lymphoid chemokines in the CNS. J Neuroimmuno 2010;224: 56-61.

37. Krumbholz M, Theil D, Cepok S, et al. Chemokines in multiple sclerosis: CXCL12 and CXCL13 up-regulation is differentially linked to CNS immune cell recruitment. Brain 2006;129:200-211.

38. Dobson R, Ramagopalan S, Davis A, Giovannoni G. Cerebrospinal fluid oligoclonal bands in multiple sclerosis and clinically isolated syndromes: a meta-analysis of prevalence, prognosis and effect of latitude. J Neurol Neurosurg Psychiatry 2013;84: 909-914.

39. Phares TW, Stohlman SA, Bergmann CC. Intrathecal humoral immunity to encephalitic RNA viruses. Viruses 2013;5:732-752.

40. Phares TW, Marques CP, Stohlman SA, Hinton DR, Bergmann CC. Factors supporting intrathecal humoral responses following viral encephalomyelitis. J Virol 2011, 85:2589-2598. 


\section{Neurology $^{\odot}$ \\ Neuroimmunology \& Neuroinflammation}

Clinical utility of a molecular signature in inflammatory demyelinating disease

Andrew R. Pachner, Krista DiSano, Darlene B. Royce, et al.

Neurol Neuroimmunol Neuroinflamm 2019;6;

DOI 10.1212/NXI.0000000000000520

This information is current as of November 9,2018

\section{Updated Information \& Services}

References

Subspecialty Collections

Permissions \& Licensing

Reprints including high resolution figures, can be found at:

http://nn.neurology.org/content/6/1/e520.full.html

This article cites 40 articles, 3 of which you can access for free at: http://nn.neurology.org/content/6/1/e520.full.html\#\#ref-list-1

This article, along with others on similar topics, appears in the following collection(s):

All Demyelinating disease (CNS)

http://nn.neurology.org//cgi/collection/all_demyelinating_disease_cns

Devic's syndrome

http://nn.neurology.org//cgi/collection/devics_syndrome

Multiple sclerosis

http://nn.neurology.org//cgi/collection/multiple_sclerosis

Transverse myelitis

http://nn.neurology.org//cgi/collection/transverse_myelitis

Information about reproducing this article in parts (figures,tables) or in its entirety can be found online at:

http://nn.neurology.org/misc/about.xhtml\#permissions

Information about ordering reprints can be found online:

http://nn.neurology.org/misc/addir.xhtml\#reprintsus

Neurol Neuroimmunol Neuroinflamm is an official journal of the American Academy of Neurology.

Published since April 2014, it is an open-access, online-only, continuous publication journal. Copyright

Copyright $\odot 2018$ The Author(s). Published by Wolters Kluwer Health, Inc. on behalf of the American

Academy of Neurology.. All rights reserved. Online ISSN: 2332-7812.

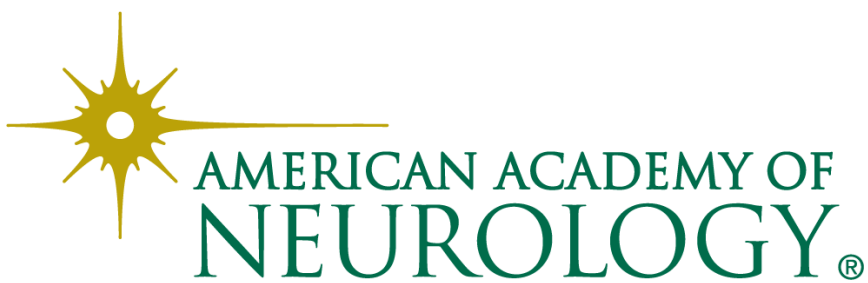

